\title{
Plasticity of Recurrent Inhibition in the Drosophila Antennal Lobe
}

\author{
Indulekha P. Sudhakaran, ${ }^{1}$ Eimear E. Holohan, ${ }^{2}$ Sahar Osman, ${ }^{2}$ Veronica Rodrigues, ${ }^{1 \dagger}$ K. VijayRaghavan, ${ }^{1}$ \\ and Mani Ramaswami ${ }^{1,2,3}$ \\ ${ }^{1}$ National Centre for Biological Sciences, Tata Institute of Fundamental Research, Bangalore 560065, India, ${ }^{2}$ School of Genetics and Microbiology and \\ School of Natural Sciences, Smurfit Institute of Genetics and Trinity College Institute of Neuroscience, Trinity College Dublin, Dublin-2, Ireland, and \\ ${ }^{3}$ Department of Molecular and Cellular Biology University of Arizona, Tucson, Arizona 85721
}

Recurrent inhibition, wherein excitatory principal neurons stimulate inhibitory interneurons that feedback on the same principal cells, occurs ubiquitously in the brain. However, the regulation and function of recurrent inhibition are poorly understood in terms of the contributing interneuron subtypes as well as their effect on neural and cognitive outputs. In the Drosophila olfactory system, odorants activate olfactory sensory neurons (OSNs), which stimulate projection neurons (PNs) in the antennal lobe. Both OSNs and PNs activate local inhibitory neurons (LNs) that provide either feedforward or recurrent/feedback inhibition in the lobe. During olfactory habituation, prior exposure to an odorant selectively decreases the animal's subsequent response to the odorant. We show here that habituation occurs in response to feedback from PNs. Output from PNs is necessary for olfactory habituation and, in the absence of odorant, direct PN activation is sufficient to induce the odorant-selective behavioral attenuation characteristic of olfactory habituation. PN-induced habituation occludes further odor-induced habituation and similarly requires GABA $\mathrm{A}_{\mathrm{A}}$ and NMDARs in PNs, as well as VGLUT and cAMP signaling in the multiglomerular inhibitory local interneurons (LN1) type of LN. Thus, PN output is monitored by an LN subtype whose resultant plasticity underlies behavioral habituation. We propose that recurrent inhibitory motifs common in neural circuits may similarly underlie habituation to other complex stimuli.

\section{Introduction}

Spatiotemporal patterns of neuronal activity, which underlie perceptual, cognitive, and behavioral outputs of nervous systems, depend on the connectivity of coupled systems of excitatory and inhibitory neurons (Buzsáki, 2010). One inhibitory motif recognized as a ubiquitous building block of neural architecture mediates recurrent or feedback inhibition. Here, activity of an excitatory principal neuron stimulates not only downstream excitatory neurons but also inhibitory interneurons that feed back onto the same principal cell to limit the duration and/or magnitude of its excitation (Isaacson and Scanziani, 2011). Most inhibitory feedback is mediated by local circuit interneurons (LNs)

\footnotetext{
Received March 6, 2012; accepted March 22, 2012.

Author contributions:I.P.S., E.E.H., V.R., K.V.R., and M.R. designed research; I.P.S. performed research; E.E.H. and S.O. contributed unpublished reagents/analytic tools; I.P.S., K.V.R., and M.R. analyzed data; I.P.S., E.E.H., V.R., K.V.R., and M.R. wrote the paper.

This work was supported by grants from Science Foundation Ireland (M.R.) and the Indian Department of Biotechnology (V.R.). I.P.S. was supported by a Government of India CSIR postgraduate fellowship. We thank Sudeshna Das and Madhumala Sadanandappa for advice and help throughout the course of these experiments, and Sive Finlay for technical assistance. We acknowledge M. Heisenberg, R. Davis, V. Jayaraman, L. Looger, L. Liu, A.-S. Chiang, G. Miesenbock, L. Vosshall, P. Garrity, T. Lee. KBasler, Rachel Wilson, and the Bloomington and Vienna Stock Centres for fly stocks and reagents. We thank Jens Hillebrand, Adrian Dervan, Aoife Larkin, John Lee, Chris Tabone, Isabell Twick, Ted Weinert, S. Sanyal, and Jing Wang for useful discussions and/or comments on the manuscript. We acknowledge Raheleh Heidari for performing the imaging experiments for Figure $2 A$. We thank David Glanzman, Alberto Ferrus, and Matthew Cobb for discussions on the characteristics of habituation.

${ }^{\dagger}$ Deceased 0ct. 11, 2011.

Correspondence should be addressed to Mani Ramaswami, Trinity College Institute of Neuroscience, Lloyd Building, Trinity College Dublin, Dublin-2 Ireland. E-mail: Mani.ramaswami@tcd.ie.

DOI:10.1523/JNEUROSCI.1099-12.2012

Copyright $\odot 2012$ the authors $\quad 0270-6474 / 12 / 327225-07 \$ 15.00 / 0$
}

that do not form long-range connections between distant brain regions. Single LNs receive excitatory inputs from a large fraction of principal cells in a given region and provide feedback inhibition onto a similar large fraction of principal cells (Fino and Yuste, 2011). While recurrent inhibition is broadly required for balancing excitation and inhibition (Lamsa et al., 2007; Kullmann and Lamsa, 2011), many aspects of its regulation and function remain to be clarified. In particular, the properties of LN subtypes that mediate recurrent inhibition as well as their roles in information processing and cognitive output are poorly understood in vivo (Bazhenov and Stopfer, 2010; Isaacson and Scanziani, 2011).

Compared with mammalian neural circuits, neurons of the insect olfactory circuit (Fig. $1 A$ ) are relatively well identified and their analysis provides insight into general coding principles and computational logic of neural circuits (Masse et al., 2009; Su et al., 2009; Bazhenov and Stopfer, 2010; Wilson, 2011). Olfactory sensory neurons (OSNs) that express identical odorant receptors project to the same glomeruli in the antennal lobe. An OSN class that mediates $\mathrm{CO}_{2}$ aversion expresses the Gr21a receptor and projects to the $\mathrm{V}$ glomerulus to contact dendrites of the $\mathrm{V}$ projection neuron (VPN). In contrast, OSN subtypes that respond to ethyl butyrate (EB) or 3-octanol express Or83b/Orco and synapse onto a distinct projection neuron (PN) class marked by GH146 promoter expression. Both OSNs and PNs either directly or indirectly stimulate inhibitory and excitatory LNs that mediate feedforward and recurrent inhibition in the antennal lobe (Fig. 1A) (Tanaka et al., 2009; Hu et al., 2010; Huang et al., 2010; Yaksi 
A

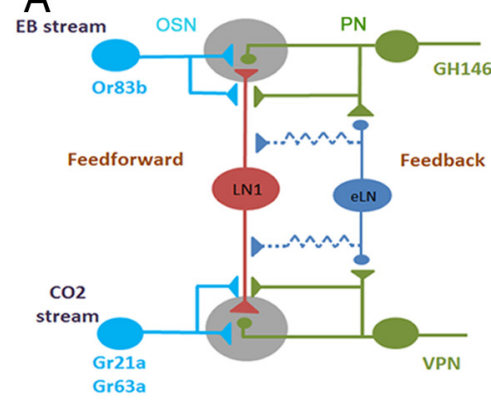

B

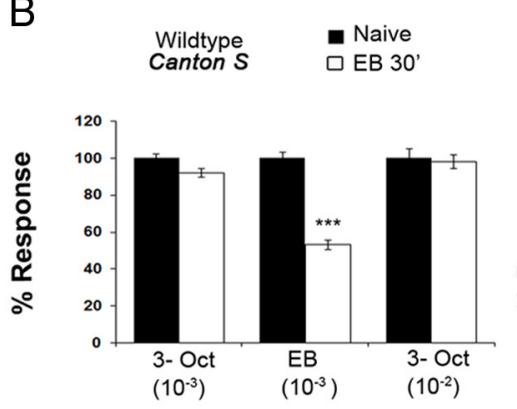

C
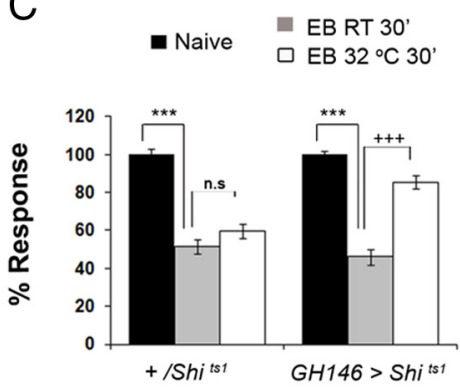

Figure 1. Activation of olfactory projection neurons is necessary for odorant-selective short-term habituation. $A$, Schematic of the Drosophila olfactory circuit showing probable feedforward (OSN-LN1) and feedback (PN-LN1-PN/PN-eLN-LN1-PN) connections. B, Response of naive and EB-exposed wild-type (Canton S) flies to EB and 3-octanol (3-0ct). EB exposure causes a selective drop in response to EB, but not to 3 -octanol at two ( $10^{-2}$ and $10^{-3}$ ) different concentrations. ${ }^{* * *} p<001$ (Student's $t$ test). $C$, EB-induced habituation in

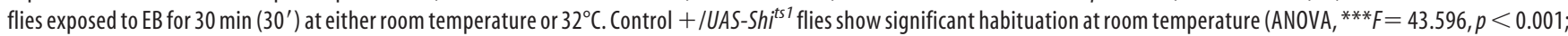
$q=12.334, p<0.001$, Student-Newman-Keuls test) and no significant difference between EB exposure at room temperature and $32^{\circ} \mathrm{C}$. In contrast, experimental flies $\mathrm{GH} 146>$ Shits flies show significant habituation at room temperature $\left({ }^{* *} F=85.016, p<0.001 ; q=17.816, p<0.001\right.$, Student-Newman-Keuls test), and a significant difference between EB exposure at room temperature and $32^{\circ} \mathrm{C}$ (when dynamin function and presynaptic transmission from GH146 PNs is inhibited, ${ }^{+++} q=13.031, p<0.001$, Student-Newman-Keuls test). Bars show mean \pm SEM. Raw RI values and $n$ values are provided in Table 1.

and Wilson, 2010). Individual LNs project to multiple glomeruli in the antennal lobe and, like analogous cells in the mammalian cortex, make inhibitory inputs onto a very large fraction of PNs (Tanaka et al., 2009; Das et al., 2011).

We recently showed that cAMP-dependent plasticity of inhibitory transmission from the multiglomerular inhibitory local interneurons (LN1) subtype of interneurons in the antennal lobe mediates olfactory habituation (Das et al., 2011), a selective reduction in behavioral response to an odorant following sustained exposure (Glanzman, 2011). We ask here whether short-term olfactory habituation (STH) is: (1) driven by recurrent inhibition and (2) mediated by its plasticity. Our findings suggest that synapse-specific potentiation of recurrent inhibition onto active PNs can account for odorant-selective habituation. We suggest that similar potentiation of recurrent inhibition, if it occurred in other brain regions, could underlie the ability of organisms to habituate to a wide range of sensory experiences.

\section{Materials and Methods}

Drosophila stocks and culture. Stocks were maintained at $25^{\circ} \mathrm{C}$ on standard corn meal agar medium unless otherwise mentioned. Gal4 and LexA stocks used are known to target specific cell types; however, these could show low levels of expression in neurons outside the targeted tissues considered. The stocks used were obtained either through stock centers or as generous gifts from the following sources: VPN-Gal4 (X) was obtained from Leslie Vosshall (Rockefeller University, New York, NY), GH146-Gal4 (II) was obtained from Reinhard Stocker (University of Fribourg, Fribourg, Switzerland), UAS- $d s N R 1$ (III) flies were from Ann-Shyn Chiang (Cold Spring Harbor Laboratory, New York, NY), UAS-RdlRNAi ${ }^{8-10 G}$ (III) was from Ron Davis (Baylor College of Medicine, Houston, TX), UAS-TRPA1 (II or III) flies were obtained from Paul Garrity (Brandeis University, Waltham/ Boston, MA), UAS-Shits ${ }^{1}$ (X and III) stock was obtained from Toshi Kitamoto (University of Iowa, Iowa City, IA), UAS-VGLUT RNAi (II) stock (VDRC 104324) and the UAS-RutRNAi (II) line (VDRC 101759) were from the Vienna Drosophila Research Centre, GH146-GAD:: LexA (II) flies were from Tzumin Lee (Janelia Farm), LexAop-TRPA1(III) flies were generated in our lab, and. shakB ${ }^{2}$ flies were obtained from Rachel Wilson (Harvard Medical School, Boston MA).

Inducing and measuring olfactory habituation. Olfactory responses were measured in sets of $\sim 25-40$ flies using the Y-maze apparatus as described previously (Das et al., 2011). The response index (RI) was defined as the fractional avoidance of an odorant-containing arm of the maze compared with a control arm containing air $\left(\mathrm{RI}=N_{\text {odor }}-\right.$ $\left.N_{\text {air }} / N_{\text {total }}\right)$. $N$ equals the number of flies. For odorant-induced habit- uation, $0-1 / 2$-d-old flies were collected and aged to $2 \mathrm{~d}$ and starved overnight on moist filter paper containing vials. The flies were pretested for their naive response to $10^{-3} \mathrm{~EB}$, then transferred to a clean glass bottle containing an Eppendorf tube with a perforated lid containing 5\% EB diluted in paraffin oil. For inducing habituation by direct PN depolarization, flies were collected and pretested for their naive response to $10^{-3} \mathrm{~EB}$ or $5 \% \mathrm{CO}_{2}$, then transferred to an incubator that maintained a constant set temperature of $29^{\circ} \mathrm{C}$ for $30 \mathrm{~min}$. The relative response of the same flies after exposure to $29^{\circ} \mathrm{C}$ was used to assess habituation. Behavioral experiments were done at room temperature $\left(22-24^{\circ} \mathrm{C}\right)$. Shak $B^{2}$ flies (and relevant controls) were reared at a temperature of $18^{\circ} \mathrm{C}$ to increase their ability to climb the Y-maze. Either ANOVA followed by Student-Newman-Keuls test or Student's $t$ test was used to estimate significance of all pairwise comparisons examined. Raw data and $n$ values for the experiments are provided in Table 1.

Conditional synaptic block experiments using UAS-Shi ${ }^{\text {ts1 }}$. For shibire experiments, the flies expressing the UAS-Shitsi transgene in appropriate cells were initially tested for response to $10^{-3} \mathrm{~EB}$ at room temperature. To inhibit synaptic output from $S h i^{t s 1}$-expressing cells during exposure, the flies were exposed for $30 \mathrm{~min}$ to $5 \% \mathrm{~EB}$ in a prewarmed bottle held in an incubator at either $32^{\circ} \mathrm{C}$ or $34^{\circ} \mathrm{C}$.

Dishabituation. For mechanically induced dishabituation, flies habituated to $\mathrm{EB} / \mathrm{CO}_{2}$ were vortexed for $1 \mathrm{~min}$ in a glass vial using a Cyclomixer (Remi Equipments). To dishabituate flies using novel olfactory stimuli, $\mathrm{CO}_{2}$-habituated flies were exposed to a strong pulse of EB by putting them for $1 \mathrm{~min}$ in a glass bottle through which vapors from a bottle containing bubbled $10^{-2}$ EB solution was passed, and EB-habituated flies were exposed to strong yeast odor in a cut-glass bottle placed on a petridish containing yeast paste. The flies were separated from the yeast by a nylon membrane.

Construction of LexAop-TrpA1 transgenic flies. The UAS-hsp70-MCSSV40polyA region (flanked by BamH1 sites) from the UAS-responding cloning vector $p U A S T a t t B$ (obtained from K. Basler, Universität Zürich, Switzerland) was excised and replaced by the LexAop-hsp70-SV40polyA region (also flanked by BamH1 sites) from the LexA-responding cloning vector LexAop (obtained from T. Lee, Janelia Farm, Ashburn, VA) to generate LexAopattB. dTRPA1 was excised from the $p O X-d T R P A 1$ vector (obtained from P. Garrity, Brandeis University, Boston, MA) by NotI/ $\mathrm{XbaI}$ restriction digest and inserted into the previously constructed LexAopattB, resulting in LexAop-TRPA1attB. LexAop-TRPAlattB was injected into the attP2 (III chromosome) landing site using $\phi$-C31 integrase (Best Gene).

GCaMP imaging to examine TRPA1-induced neuronal excitation. The Drosophila brain preparation and imaging techniques were as described previously (Das et al., 2011). GCaMP-expressing cells were first imaged 
Table 1. Raw RI of all genotypes in different experimental conditions

\begin{tabular}{|c|c|c|c|}
\hline Genotype & Exposure & Pretest response $(n)$ [to odor] & Posttest response $(n)$ [to odor] \\
\hline \multirow[t]{3}{*}{ CS } & $5 \% \mathrm{~EB}$ & $0.73 \pm 0.02(13)\left[10^{-3} 30 \mathrm{ct}\right]$ & $0.68 \pm 0.02(13)\left[10^{-3} 30 \mathrm{ct}\right]$ \\
\hline & $5 \% \mathrm{~EB}$ & $0.51 \pm 0.01(9)\left[10^{-2} 30 \mathrm{ct}\right]$ & $0.50 \pm 0.01(9)\left[10^{-2} 30 \mathrm{ct}\right]$ \\
\hline & $5 \% \mathrm{~EB}$ & $0.71 \pm 0.02(18)\left[10^{-3} \mathrm{~EB}\right]$ & $0.38 \pm 0.01(18)\left[10^{-3} \mathrm{~EB}\right]$ \\
\hline Shak $B^{2}$ & $5 \%$ EB & $0.66 \pm 0.02(9)\left[10^{-3} \mathrm{~EB}\right]$ & $0.50 \pm 0.03(9)\left[10^{-3} \mathrm{~EB}\right]$ \\
\hline \multirow[t]{2}{*}{$C S>S h i t s 1$} & $5 \% \mathrm{~EB}$ at $25^{\circ} \mathrm{C}$ & $0.66 \pm 0.02(8)\left[10^{-3} \mathrm{~EB}\right]$ & $0.34 \pm 0.02(8)\left[10^{-3} \mathrm{~EB}\right]$ \\
\hline & $5 \% \mathrm{~EB}$ at $32^{\circ} \mathrm{C}$ & & $0.39 \pm 0.02(8)\left[10^{-3} \mathrm{~EB}\right]$ \\
\hline \multirow[t]{2}{*}{ GH146 $>S h i^{t 51}$} & $5 \% \mathrm{~EB}$ at $25^{\circ} \mathrm{C}$ & $0.65 \pm 0.01(9)\left[10^{-3} \mathrm{~EB}\right]$ & $0.30 \pm 0.02(5)\left[10^{-3} \mathrm{~EB}\right]$ \\
\hline & $5 \% \mathrm{~EB}$ at $32^{\circ} \mathrm{C}$ & & $0.56 \pm 0.02(9)\left[10^{-3} \mathrm{~EB}\right]$ \\
\hline \multirow[t]{2}{*}{$C S>S h i t s 1$} & $5 \% \mathrm{~EB}$ at $25^{\circ} \mathrm{C}$ & $0.68 \pm 0.02(4)\left[10^{-3} \mathrm{~EB}\right]$ & $0.30 \pm 0.02(8)\left[10^{-3} \mathrm{~EB}\right]$ \\
\hline & $5 \% \mathrm{~EB}$ at $32^{\circ} \mathrm{C}$ & & $0.34 \pm 0.02(8)\left[10^{-3} \mathrm{~EB}\right]$ \\
\hline \multirow[t]{2}{*}{$M B 247>S h i^{t 51}$} & $5 \% \mathrm{~EB}$ at $25^{\circ} \mathrm{C}$ & $0.69 \pm 0.02(7)\left[10^{-3} \mathrm{~EB}\right]$ & $0.32 \pm 0.04(6)\left[10^{-3} \mathrm{~EB}\right]$ \\
\hline & $5 \% \mathrm{~EB}$ at $32^{\circ} \mathrm{C}$ & & $0.39 \pm 0.03(10)\left[10^{-3} \mathrm{~EB}\right]$ \\
\hline GH146 > TRPA1 & $29^{\circ} \mathrm{C}$ & $0.72 \pm 0.03(10)\left[10^{-3} \mathrm{~EB}\right]$ & $0.33 \pm 0.04(10)\left[10^{-3} \mathrm{~EB}\right]$ \\
\hline$V P N>T R P A 1$ & $29^{\circ} \mathrm{C}$ & $0.65 \pm 0.03(11)\left[10^{-3} \mathrm{~EB}\right]$ & $0.57 \pm 0.02(11)\left[10^{-3} \mathrm{~EB}\right]$ \\
\hline GH146 > TRPA1/dsNR1RNAi & $29^{\circ} \mathrm{C}$ & $0.74 \pm 0.03(8)\left[10^{-3} \mathrm{~EB}\right]$ & $0.65 \pm 0.05(9)\left[10^{-3} \mathrm{~EB}\right]$ \\
\hline GH146> TRPA1/RdIRNAi & $29^{\circ} \mathrm{C}$ & $0.64 \pm 0.01(9)\left[10^{-3} \mathrm{~EB}\right]$ & $0.54 \pm 0.02(10)\left[10^{-3} \mathrm{~EB}\right]$ \\
\hline GH146,LN1 > TRPAp1 & $29^{\circ} \mathrm{C}$ & $0.68 \pm 0.02(10)\left[10^{-3} \mathrm{~EB}\right]$ & $0.37 \pm 0.02(7)\left[10^{-3} \mathrm{~EB}\right]$ \\
\hline GH146,LN1 > VGLUTi;TRPA1 & $29^{\circ} \mathrm{C}$ & $0.69 \pm 0.02(9)\left[10^{-3} \mathrm{~EB}\right]$ & $0.60 \pm 0.02(10)\left[10^{-3} \mathrm{~EB}\right]$ \\
\hline GH146,LN1 > RutRNAi;TRPA1 & $29^{\circ} \mathrm{C}$ & $0.67 \pm 0.01(10)\left[10^{-3} \mathrm{~EB}\right]$ & $0.56 \pm 0.01(11)\left[10^{-3} \mathrm{~EB}\right]$ \\
\hline ShakB ${ }^{2} ; G H 146>$ TRPA1 & $29^{\circ} \mathrm{C}$ & $0.65 \pm 0.04(9)\left[10^{-3} \mathrm{~EB}\right]$ & $0.58 \pm 0.03(9)\left[10^{-3} \mathrm{~EB}\right]$ \\
\hline$V P N>T R P A 1$ & $29^{\circ} \mathrm{C}$ & $0.70 \pm 0.03(8)\left[5 \% \mathrm{CO}_{2}\right]$ & $0.30 \pm 0.02(9)\left[5 \% \mathrm{CO}_{2}\right]$ \\
\hline GH146 $>$ TRPA1 & $29^{\circ} \mathrm{C}$ & $0.68 \pm 0.03(9)\left[5 \% \mathrm{CO}_{2}\right]$ & $0.61 \pm 0.05(10)\left[5 \% \mathrm{CO}_{2}\right]$ \\
\hline$V P N>T R P A 1 ; d s N R 1 R N A i$ & $29^{\circ} \mathrm{C}$ & $0.64 \pm 0.02(10)\left[5 \% \mathrm{CO}_{2}\right]$ & $0.57 \pm 0.02(10)\left[5 \% \mathrm{CO}_{2}\right]$ \\
\hline$V P N>T R P A 1 ; R d I R N A i$ & $29^{\circ} \mathrm{C}$ & $0.70 \pm 0.02(11)\left[5 \% \mathrm{CO}_{2}\right]$ & $0.60 \pm 0.04(12)\left[5 \% \mathrm{CO}_{2}\right]$ \\
\hline \multirow[t]{3}{*}{$+/ T R P A 1$} & $5 \% \mathrm{~EB}$ & $0.58 \pm 0.02(9)\left[10^{-3} \mathrm{~EB}\right]$ & $0.26 \pm 0.01(8)\left[10^{-3} \mathrm{~EB}\right]$ \\
\hline & $29^{\circ} \mathrm{C}$ & $0.60 \pm 0.03(8)\left[10^{-3} \mathrm{~EB}\right]$ & $0.56 \pm 0.03(9)\left[10^{-3} \mathrm{~EB}\right]$ \\
\hline & $5 \% \mathrm{~EB}+29^{\circ} \mathrm{C}$ & $0.64 \pm 0.04(5)\left[10^{-3} \mathrm{~EB}\right]$ & $0.30 \pm 0.03(9)\left[10^{-3} \mathrm{~EB}\right]$ \\
\hline \multirow[t]{3}{*}{ GH146 $>$ TRPA1 } & $5 \% \mathrm{~EB}$ & $0.60 \pm 0.02(8)\left[10^{-3} \mathrm{~EB}\right]$ & $0.26 \pm 0.02(7)\left[10^{-3} \mathrm{~EB}\right]$ \\
\hline & $29^{\circ} \mathrm{C}$ & $0.57 \pm 0.03(9)\left[10^{-3} \mathrm{~EB}\right]$ & $0.30 \pm 0.03(9)\left[10^{-3} \mathrm{~EB}\right]$ \\
\hline & $5 \% \mathrm{~EB}+29^{\circ} \mathrm{C}$ & $0.61 \pm 0.04(10)\left[10^{-3} \mathrm{~EB}\right]$ & $0.23 \pm 0.03(10)\left[10^{-3} \mathrm{~EB}\right]$ \\
\hline \multirow[t]{3}{*}{ +/TRPA1 } & $15 \% \mathrm{CO}_{2}$ & $0.70 \pm 0.02(6)\left[5 \% \mathrm{CO}_{2}\right]$ & $0.36 \pm 0.01(6)\left[5 \% \mathrm{CO}_{2}\right]$ \\
\hline & $29^{\circ} \mathrm{C}$ & $0.64 \pm 0.01(8)\left[5 \% \mathrm{CO}_{2}\right]$ & $0.61 \pm 0.02(7)\left[5 \% \mathrm{CO}_{2}\right]$ \\
\hline & $15 \% \mathrm{CO}_{2}+29^{\circ} \mathrm{C}$ & $0.62 \pm 0.02(8)\left[5 \% \mathrm{CO}_{2}\right]$ & $0.28 \pm 0.03(8)\left[5 \% \mathrm{CO}_{2}\right]$ \\
\hline \multirow[t]{3}{*}{$V P N>T R P A 1$} & $15 \% \mathrm{CO}_{2}$ & $0.67 \pm 0.02(6)\left[5 \% \mathrm{CO}_{2}\right]$ & $0.40 \pm 0.01(8)\left[5 \% \mathrm{CO}_{2}\right]$ \\
\hline & $29^{\circ} \mathrm{C}$ & $0.58 \pm 0.01(8)\left[5 \% \mathrm{CO}_{2}\right]$ & $0.36 \pm 0.01(9)\left[5 \% \mathrm{CO}_{2}\right]$ \\
\hline & $15 \% \mathrm{CO}_{2}+29^{\circ} \mathrm{C}$ & $0.61 \pm 0.02(10)\left[5 \% \mathrm{CO}_{2}\right]$ & $0.36 \pm 0.03(11)\left[5 \% \mathrm{CO}_{2}\right]$ \\
\hline \multirow[t]{3}{*}{ GH146 > TRPA1 } & $29^{\circ} \mathrm{C}$ & $0.63 \pm 0.03(10)\left[10^{-3} \mathrm{~EB}\right]$ & $0.30 \pm 0.02(9)\left[10^{-3} \mathrm{~EB}\right], 0 \mathrm{~h}$ \\
\hline & & & $0.48 \pm 0.02(9)\left[10^{-3} \mathrm{~EB}\right], 1 \mathrm{~h}$ \\
\hline & & & $0.56 \pm 0.03(9)\left[10^{-3} \mathrm{~EB}\right], 3.5 \mathrm{~h}$ \\
\hline \multirow[t]{3}{*}{$V P N>T R P A 1$} & $29^{\circ} \mathrm{C}$ & $0.59 \pm 0.02(9)\left[5 \% \mathrm{CO}_{2}\right]$ & $0.19 \pm 0.01(8)\left[5 \% \mathrm{CO}_{2}\right], 0 \mathrm{~h}$ \\
\hline & & & $0.36 \pm 0.02(8)\left[5 \% \mathrm{CO}_{2}\right], 1 \mathrm{~h}$ \\
\hline & & & $0.52 \pm 0.03(8)\left[5 \% \mathrm{CO}_{2}\right], 3.5 \mathrm{~h}$ \\
\hline \multirow[t]{2}{*}{ GH146 $>$ TRPA1 } & $29^{\circ} \mathrm{C}$ & $0.60 \pm 0.02(5)\left[10^{-3} \mathrm{~EB}\right]$ & $0.24 \pm 0.03(7)\left[10^{-3} \mathrm{~EB}\right]$ \\
\hline & $29^{\circ} \mathrm{C}+$ vortexing & & $0.55 \pm 0.02(8)\left[10^{-3} \mathrm{~EB}\right]$ \\
\hline \multirow[t]{2}{*}{$V P N>T R P A 1$} & $29^{\circ} \mathrm{C}$ & $0.63 \pm 0.04(7)\left[5 \% \mathrm{CO}_{2}\right]$ & $0.29 \pm 0.02(8)\left[5 \% \mathrm{CO}_{2}\right]$ \\
\hline & $29^{\circ} \mathrm{C}+$ vortexing & & $0.56 \pm 0.03(8)\left[5 \% \mathrm{CO}_{2}\right]$ \\
\hline \multirow[t]{2}{*}{ GH146 > TRPA1 } & $29^{\circ} \mathrm{C}$ & $0.70 \pm 0.04(6)\left[10^{-3} \mathrm{~EB}\right]$ & $0.40 \pm 0.03(6)\left[10^{-3} \mathrm{~EB}\right]$ \\
\hline & $29^{\circ} \mathrm{C}+$ yeast puff & & $0.59 \pm 0.03(6)\left[10^{-3} \mathrm{~EB}\right]$ \\
\hline \multirow{2}{*}{$V P N>T R P A 1$} & $29^{\circ} \mathrm{C}$ & $0.62 \pm 0.02(6)\left[5 \% \mathrm{CO}_{2}\right]$ & $0.28 \pm 0.01(6)\left[5 \% \mathrm{CO}_{2}\right]$ \\
\hline & $29^{\circ} \mathrm{C}+$ EB pulse & & $0.56 \pm 0.03(6)\left[5 \% \mathrm{CO}_{2}\right]$ \\
\hline
\end{tabular}

$n$ is the number of sets done for each condition; values are \pm SEM. 30 ct, 3-0ctanol.

in a peltier-controlled dish at $22^{\circ} \mathrm{C}$. The saline was then warmed to $28^{\circ} \mathrm{C}$ within $2 \mathrm{~min}$ and the cells imaged again.

\section{Results}

PN output is necessary for odorant-selective olfactory habituation

Drosophila exposed to either $5 \% \mathrm{~EB}$ or $15 \% \mathrm{CO}_{2}$ for 30 min show a selectively reduced response to the respective odorant for approximately an hour after exposure (Das et al., 2011). This selectivity of STH is apparent not only between $\mathrm{EB}$ and $\mathrm{CO}_{2}$ (Das et al., 2011), an atypical odorant conveniently sensed by a distinct glomerulus, but also between EB and 3-octanol (Fig. $1 B$ ). Thus, flies exposed to 5\% $\mathrm{EB}$ for $30 \mathrm{~min}$ show no decrement in their response to 3-octanol
(Fig. $1 B$ ). If excitation from OSNs were sufficient to drive habituation, then habituation would not require synaptic output from PNs during initial odorant exposure. To test this hypothesis, we conditionally blocked output from the EB-responsive GH146 subset of PNs, engineered to express a dominant, conditionally mutant form of dynamin (Shi) (Kitamoto, 2001). UAS-Shits1 /+;GH146-Gal4/+; $U A S-S h i^{t s 1} /+$ flies showed normal STH when exposed to EB at room temperature $\left(21-24^{\circ} \mathrm{C}\right)$, when dynamin-dependent synaptic vesicle recycling should occur normally. However, these same flies did not habituate when exposed at $32^{\circ} \mathrm{C}$, a temperature at which dynamin function would be inhibited (Fig. 1C). Thus, neurotransmitter release from excitatory projection neurons is necessary for 
A

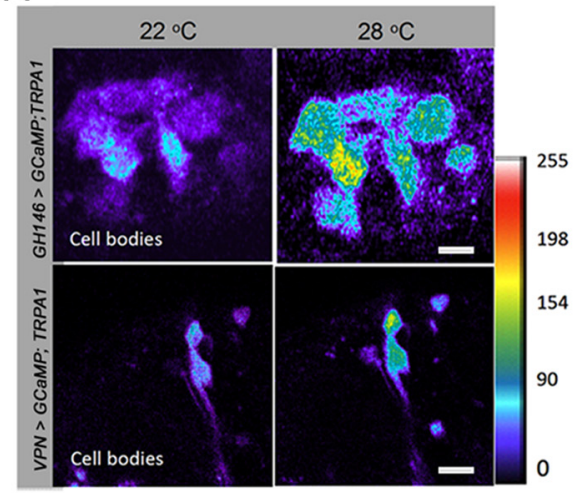

C

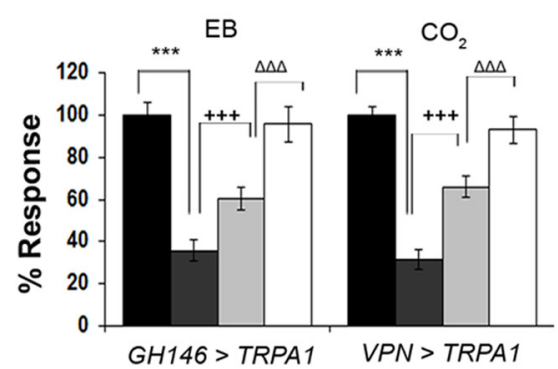

$A^{\prime}$
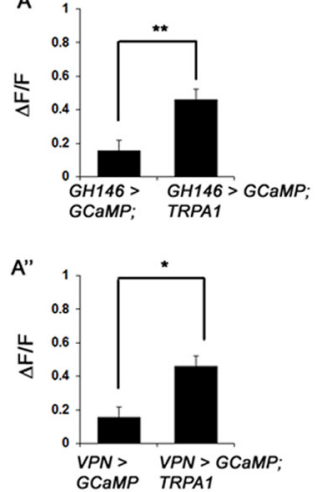

D

B
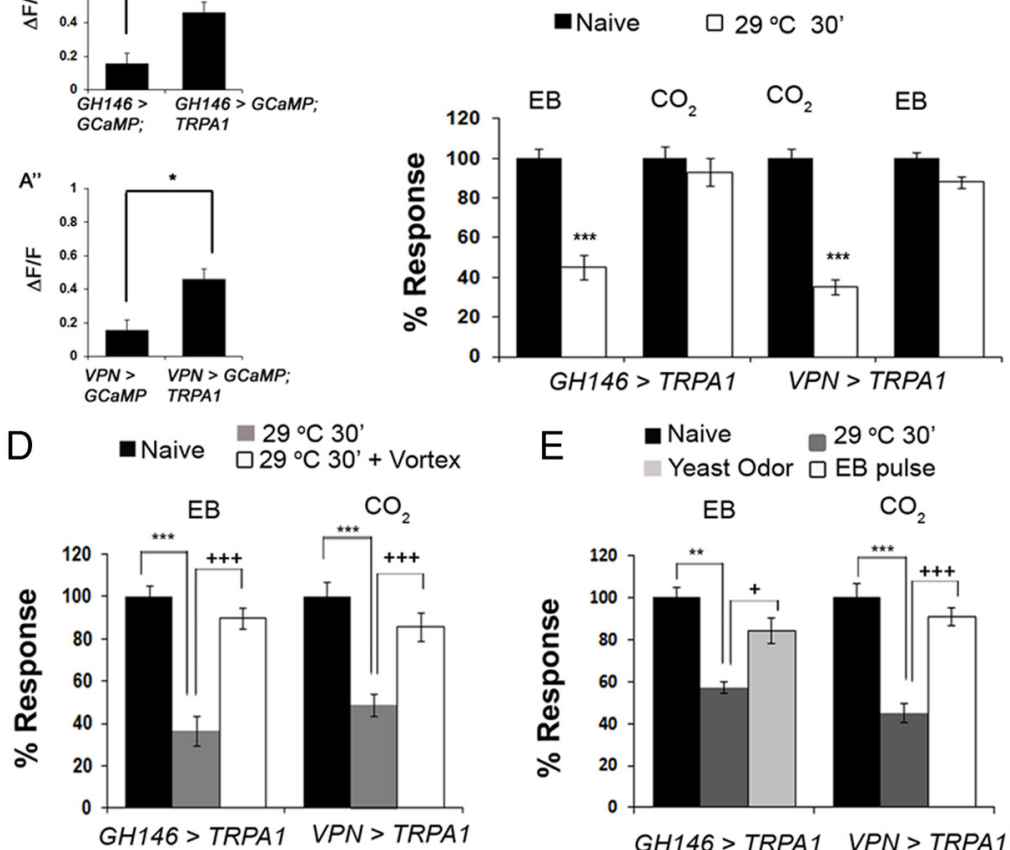

$\mathrm{CO}_{2}$

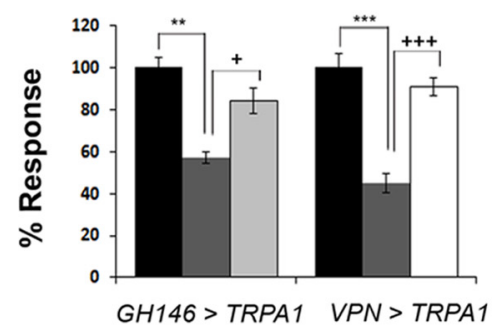

Figure 2. PNactivation is sufficient to induce odorant-selective habituation. $A, G C a M P$ imaging to measure temperature-induced calcium fluxes in TrpA1-expressing projection neurons in the antennal lobe.

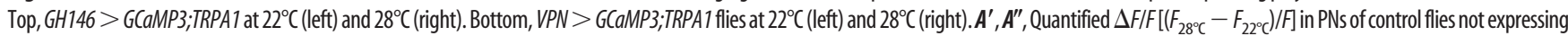
TRPA1 (GH146 > GCAMP3 and VPN > GCAMP3) compared, respectively, to flies expressing TRPA1 (GH146> GCaMP3, TRPA1; $\left.\boldsymbol{A}^{\prime},{ }^{* *} p<0.01\right)$ and VPN1 $>$ GCAMP3, TRPA1 ( $\left.\boldsymbol{A}^{\prime \prime},{ }^{*} p<0.05\right)$. B, 0dorantselective habituation induced by direct activation of cognate PNs using TRPA1. GH146 $>$ TRPA1 show normal STH to EB but not to $\mathrm{CO}_{2}$; VPN $>$ TRPA1 show normal STH to $\mathrm{CO}_{2}$ but not to EB. ${ }^{* * *} p<0.001$ (Student's $t$ test). C, Time dependence of PN-induced habituation to either $\mathrm{EB}$ or $\mathrm{CO}_{2}$. ANOVA shows significant difference in response between naive and $29^{\circ} \mathrm{C}$-exposed responses for $\mathrm{GH} 146>$ TRPA1 flies $\left({ }^{* * *} F=85.321, p<0.001, q=20.075, p<0.001\right.$, Student-Newman-Keuls test) and for VPN $>$ TRPA1flies $\left({ }^{* * *} F=79.794, p<0.001, q=19.598, p<0.001\right.$, Student-Newman-Keuls test). Posthoc testing shows a significant difference in $\mathrm{EB} / \mathrm{CO}_{2}$ response between 0 - and 1-h-recovered GH146 $>$ TRPA1 flies $\left({ }^{++}{ }^{+} q=5.4, p<0.001\right.$ vs $\left.q=6.885, p<0.001\right)$ and VPN $>$ TRPA1 flies $\left({ }^{++}+{ }^{+} q=2.6885\right.$, $p<0.001) . E B / C_{2}$ response of 1- and 3.5-h-recovered showed significant difference for GH146>TRPA1 flies $\left({ }^{\Delta \Delta \Delta} q=10.291, p<0.001\right)$ and VPN $>\operatorname{TRPA1}$ flies $\left({ }^{\Delta \Delta \Delta} q=9.397, p<0.001\right) . \boldsymbol{D}$, Mechanically induced dishabituation from GH146-induced habituation (left) and VPN-induced habituation (right). ANOVA showed a significant difference in response between naive and $29^{\circ} \mathrm{C}$ exposed flies for GH146 $>$ TRPA1 flies ( ${ }^{* * *} F=75.591, p<0.001, q=15.978, p<0.001$, Student-Newman-Keuls test) and for VPN $>$ TRPA1 flies $\left({ }^{* * *} F=90.154, p<0.001, q=18.144, p<0.001\right.$, Student-NewmanKeuls test). Posthoctesting of $29^{\circ}$ Cexposed flies versus $29^{\circ}$ Cexposed flies that were also vortexed showed a significant difference in response $\left(^{+++} q=13.930, p<0.001\right.$ for $G H 146>\operatorname{TRPA} 1$ and $q=13.926$, $p<0.001$ for VPN $>$ TRPA1/+).E, Dishabituation of PN (GH146)-induced habituation to EB habituation by exposure to strong yeast odor (left) or VPN-induced $\mathrm{CO}_{2}$ habituation by exposure to $10^{-2}$ EB pulse (right). ANOVA showed a significant difference in response between naive and $29^{\circ}$ Cexposed flies for $G H 146>\operatorname{TRPA}$ flies $\left({ }^{* *} F=14.538, p<0.003, q=7.457, p<0.003\right.$, Student-Newman-Keuls test) and for VPN $>$ TRPA1 flies $\left({ }^{* * *} F=21.636, p<0.001, q=9.215, p<0.001\right.$, Student-Newman-Keuls test). Post hoc testing of $29^{\circ} \mathrm{C}$ exposed flies versus $29^{\circ} \mathrm{C}$ exposed flies that were later exposed to either yeast odor (GH146 $>$ TRPA1) or an EB pulse (VPN > TRPA1) showed a significant difference in response $\left({ }^{+} q=3.857, p<0.03\right)$ for GH146 $>$ TRPA1 and $\left({ }^{+++} q=7.154, p<0.001\right)$ for VPN $>$ TRPA1. Bars show mean \pm SEM. Raw RI and $n$ values for are provided in Table 1.

inducing habituation, indicating that OSN-LN excitation on its own cannot drive plasticity processes that underlie habituation.

PN output is sufficient for odorantselective olfactory habituation To further investigate whether OSN-LN excitation was at all necessary for inducing olfactory habituation, we tested whether direct PN depolarization using transgenically expressed TRPA1 channels would be sufficient to cause the reduced olfactory response typical of olfactory habituation. TRPA1 channels are quiescent at temperatures $<25^{\circ} \mathrm{C}$, but open to form cationpermeable channels at higher temperatures (Rosenzweig et al., 2005; Pulver et al., 2009). Thus, warming GH146-Gal4/UAS-TRPA1 or $V P N-G a l 4 /+; U A S-T R P A 1 /+$ flies to
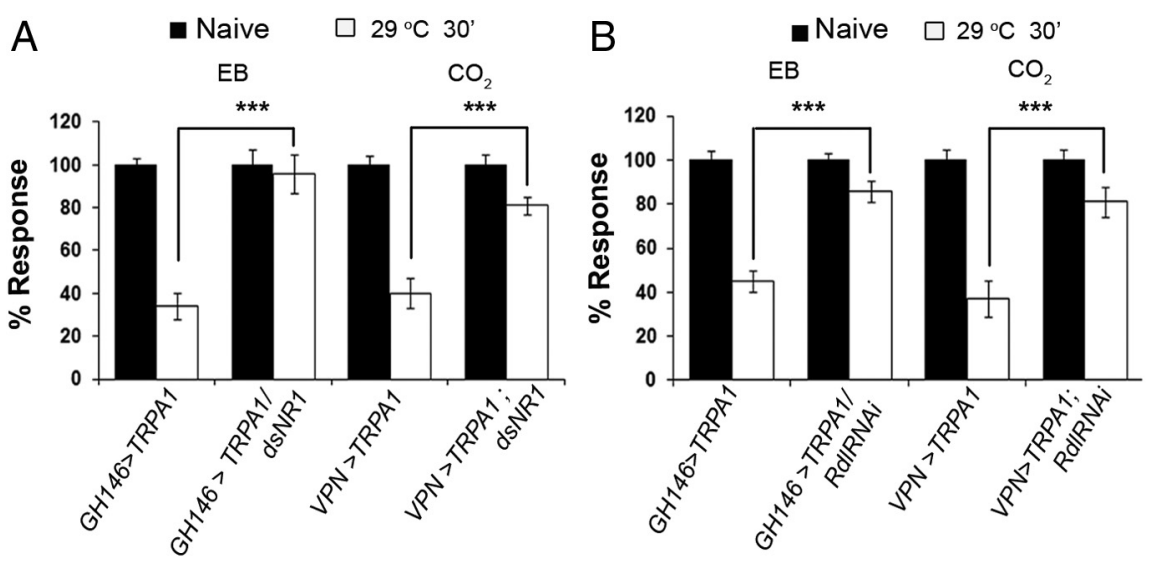

Figure 3. $G A B A_{A}$ - and NMDA-type glutamate receptors are required in PNs for PN-induced habituation. $A, B, P N$-induced habituation in flies with PN-specific knockdown of glutamate receptor NMDAR $(\boldsymbol{A})$ or $G A B A_{A}$ receptor Rdl $(\boldsymbol{B})$ using previously validated UAS-RNAi transgenes. The VPN $>$ TRPA1 and GH146 $>$ TRPA1 flies, which normally display an attenuated response to $\mathrm{CO}_{2}$ or EB, respectively, after 30 min exposure to $29^{\circ} \mathrm{C}$, do not show significantly changed responses if NMDAR or Rdl are knocked down in respective PNs. ${ }^{* * *} p<0.001$ (Student's $t$ test). Bars show mean \pm SEM. Raw RI and $n$ values are provided in Table 1. 
$>28^{\circ} \mathrm{C}$ causes direct activation of the targeted subset of PNs, as shown by GCaMP imaging of these neurons (Fig. $2 \mathrm{~A}$ ).

Thirty-minute exposure of GH146-Gal4/UAS-TRPA1 flies to $29^{\circ} \mathrm{C}$ was sufficient to induce a reduction in the avoidance response to $\mathrm{EB}$ but not to $\mathrm{CO}_{2}$ (Fig. $2 \mathrm{~B}$ ). In contrast, similar activation of VPNs that project to the $\mathrm{CO}_{2}$-responsive $\mathrm{V}$ glomerulus resulted in reduced olfactory avoidance to $\mathrm{CO}_{2}$ but had no effect on the EB response (Fig. $2 \mathrm{~B}$ ). These data show that in the complete absence of OSN stimulation, PN activation is sufficient to induce a state that resembles odorant-induced olfactory habituation (Larkin et al., 2010; Das et al., 2011). Similar to odorantinduced habituation, the $\mathrm{PN}$-induced reduced olfactory avoidance response recovered substantially after $1 \mathrm{~h}$ at room temperature (Fig. 2C). In addition, this learned reduced avoidance could be erased either by brief, intense mechanical stimulation (Fig. 2D) or by exposure to strong novel odorants (Fig. 2E). Thus, $\mathrm{PN}$-induced habituation shows the basic characteristics of habituation: it is odor-selective, recovers over time, and, most critically, is subject to dishabituation by different classes of dishabituating stimuli (Thompson and Spencer, 1966; Rankin et al., 2009). Moreover, the observed phenomenon of dishabituation indicates that the reduced avoidance response is not due to neuronal fatigue, which could potentially arise from prolonged $\mathrm{PN}$ stimulation.

The above experiments indicate that the odor-induced activation of OSNs is neither necessary nor sufficient to induce behavioral habituation; in contrast, activation of PNs is both necessary and sufficient to induce a habituated state. Given that OSN activation induces habituation by potentiating inhibitory LN transmission in the antennal lobe (Das et al., 2011), the above data point to a model in which habituation is driven by feedback from PNs to LNs.

However, an alternative possibility is that $\mathrm{PN}$-induced habituation occurs through a distinct mechanism that involves circuit elements downstream of the antennal lobe. We therefore performed additional experiments to ask whether odorant- and $\mathrm{PN}$-induced habituation occurred through similar circuit mechanisms.

\section{PN feedback to LNs underlies olfactory habituation}

Both EB- and $\mathrm{CO}_{2}$-induced habituation require NMDARs and $\mathrm{GABA}_{\mathrm{A}}(\mathrm{Rdl})$ receptor function in PNs as well as the Rutabaga adenylate cyclase, required for many forms of habituation in Drosophila (Corfas and Dudai, 1989; Engel and Wu, 1996), and vesicular glutamate transporter (VGLUT) in LNs (Das et al., 2011). If $\mathrm{PN}$-induced habituation occurs through the same circuit mechanism, then we expected it to have similar molecular and anatomical requirements.

Knockdown of either NMDARs or Rdl receptors in PNs using previously validated transgenic RNAi constructs had the same effect on PN-induced habituation as they did on odorantinduced habituation. Knockdown of the NMDAR subunit NR1 through expression of UAS-dsNR1 in either the GH146 or VPN subset of PNs expressing TRPA1 caused an odorant-selective block in PN-induced habituation (Xia et al., 2005; Wu et al., 2007; Das et al., 2011). Thus, while $30 \mathrm{~min}$ of exposure to $29^{\circ} \mathrm{C}$ reduced the $\mathrm{CO}_{2}$ avoidance of VPN-Gal4/+;UAS-TRPA1/+ flies, it had no effect on the $\mathrm{CO}_{2}$ avoidance of VPN-Gal4/+;UAS-TRPA1/+; $U A S-d s N R 1 /+$ animals. Similarly, the reduced EB avoidance of GH146-Gal4/+; UAS-TRPA1/+ flies observed after heat exposure was not evident in GH146-Gal4/+; UAS-TRPA1/UAS$d s N R 1$ flies (Fig. 3A). Knockdown of $\mathrm{GABA}_{\mathrm{A}}$ receptors by targeted expression of a UAS-RdlRNAi construct (Liu et al., 2007, 2009; Das et al., 2011) in PNs expressing TRPA1 also similarly
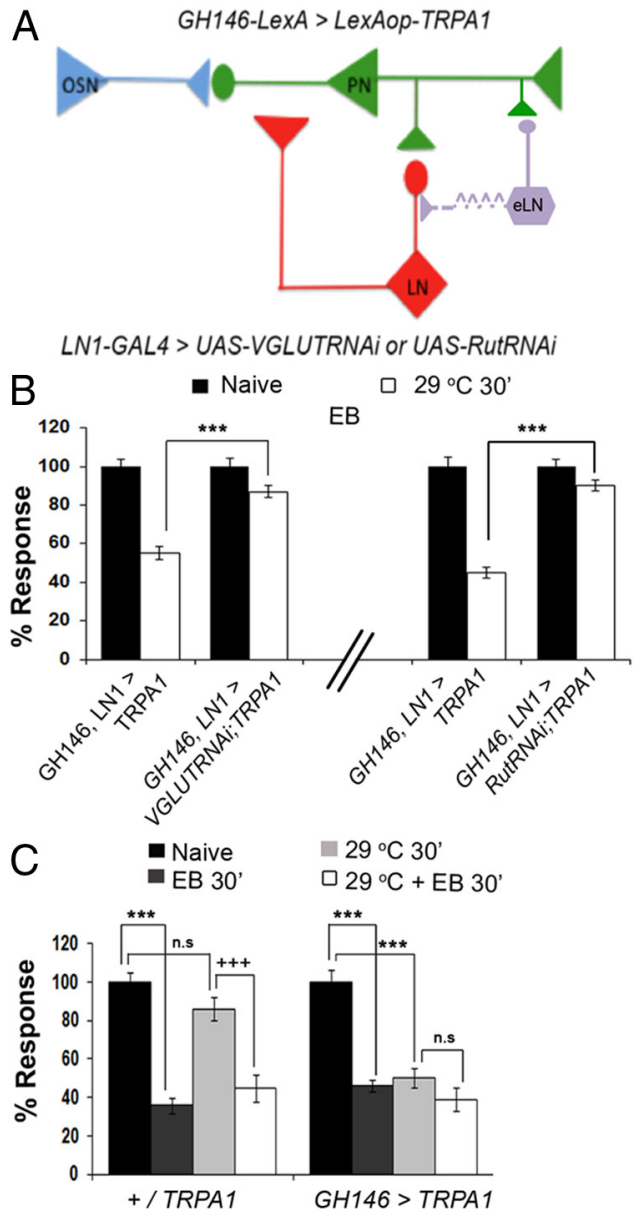

Figure 4. Use of a dual binary transcriptional system to show that CAMP signaling and vesicular glutamate release in $\mathrm{LN}$ s is required for $\mathrm{PN}$-induced habituation. Habituation observed by EB exposure or GH146-PN activation are not additive, suggesting a shared circuit mechanism. A, Schematic showing how expression of TRPA1 in PNs by combining GH146-GAD::LexA and LexAop-TRPA1 transgenes allows one to simultaneously use the Gal4 system to express UASRNAi transgenes in LNs. $\boldsymbol{B}, G H 146, L N 1>$ TRPA1 flies show habituation to EB after exposure to $29^{\circ} \mathrm{C}$ for 30 min while the GH146, LN1 > vGLUTRNAi;TRPA1 and GH146, LN1 > RutRNAi; TRPA1 flies do not. ${ }^{* *} p<0.001$ (Student's $t$ test). C, In GH146 $>$ TRPA1 flies, exposure to either $5 \%$ $\mathrm{EB}$, to $29^{\circ} \mathrm{C}$, or to both causes similar levels of habituation. For control +/TRPA1 flies, ANOVA shows significant difference between naive and EB-exposed flies $\left({ }^{* * *} F=67.218, p<0.001\right.$; $q=14.389, p<0.001$, Student-Newman-Keuls test), but no significant difference between naive and $29^{\circ} \mathrm{C}$-exposed flies. $\mathrm{EB}+29^{\circ} \mathrm{C}$ and $29^{\circ} \mathrm{C}$ alone exposed flies also differ significantly $\left({ }^{++} q=13.510, p<0.001\right)$ but show no significant difference between $\mathrm{EB}+29^{\circ} \mathrm{C}$ and $\mathrm{EB}$ alone. GH146 $>$ TRPA1 flies show a significant difference in response following either EBexposure $\left({ }^{* * *} F=46.593, p<0.001 ; q=12.688, p<0.001\right.$, Student-Newman-Keuls test), or $29^{\circ} \mathrm{C}$ exposure $\left.{ }^{* * *} q=14.074, p<0.001\right)$. No significant difference between $\mathrm{EB}+29^{\circ} \mathrm{C}$ and $\mathrm{EB}$-exposure alone or between $\mathrm{EB}+29^{\circ} \mathrm{C}$ and $29^{\circ} \mathrm{C}$ exposure was seen. Bars show \pm mean SEM. Raw RI and $n$ values are shown in Table 1.

blocked habituation induced by exposure to $29^{\circ} \mathrm{C}$ for $30 \mathrm{~min}$ (Fig. $3 B)$. In contrast, expression of a control UAS-AchRGFP transgene had no effect on $\mathrm{PN}$-induced habituation. Thus, neurotransmitter receptors for glutamate and $\mathrm{GABA}_{\mathrm{A}}$ are required in PNs for both odor- and $\mathrm{PN}$-induced habituation. These data argue that $\mathrm{PN}$-induced habituation depends on GABAergic and glutamatergic signaling inputs onto PNs, probably in the antennal lobe where all currently known inputs to PNs reside.

To more definitively establish that PN-induced habituation occurs by recruiting a recurrent inhibitory pathway involving LN1 neurons of the antennal lobe, we created transgenes that allowed use of dual binary transcription systems: one system to allow direct depolarization of the PNs, and the other to simulta- 
neously knockdown either rut or VGLUT required in LNs for odor-induced habituation (Fig. 4A) (Lai and Lee, 2006; Das et al., 2011).

Expression of a LexA-responsive TRPA1 transgene (LexAop-TRPA1) in PNs driven by GH146-GAD::LexA (Lai and Lee, 2006) allowed the establishment of $\mathrm{PN}$-induced EB habituation. However. this habituation was greatly reduced when validated RNAi constructs against either VGLUT or rutabaga activity were expressed in the LN1 class of local interneurons (Das et al., 2011). Thus, while GH146-GAD::LexA,LN1-Gal4/+; LexAop-TRPA1/+ flies show reduced EB avoidance after $30 \mathrm{~min}$ of exposure to $29^{\circ} \mathrm{C}$, identically treated GH146-GAD::LexA, LN1-Gal4/UAS-VGLUTRNAi; LexAopTRPA1/+ and GH146-GAD::LexA,LN1-GAL4/

UAS-RutRNAi;LexAop-TRPA1/+ flies did not show an altered EB response (Fig. $4 B$ ). Thus, $\mathrm{PN}$-induced habituation, with similar signaling requirements in both PNs and LNs, has the same molecular and anatomical requirements as odorant-induced habituation.

Together, these experiments establish that direct PN activation induces habituation by recruiting LNs, which in turn participate in cAMP and NMDAR-dependent plasticity of LN-PN synapses necessary for $\mathrm{PN}$-induced habituation. The second major conclusion that odorant- and $\mathrm{PN}$-induced habituation occur through the same circuit mechanism is supported by three lines of evidence: (1) odor-induced habituation requires output from $\mathrm{PNs}$, which is inconsistent with an OSN-LN pathway for habituation; (2) both require identical signaling components in LNs and PNs; and (3) there is no additive effect when odor exposure and $\mathrm{PNs}$ activation are combined (Fig. 4C).

\section{ShakB-encoded electrical synapses are required for olfactory habituation}

Recurrent inhibition from PNs to LN1 may occur either through a direct excitation, or through an indirect pathway mediated through either a recently identified centrifugal feedback pathway from the mushroom body (MB) to LNs (Hu et al., 2010), or via excitatory LNs (eLNs) in the lobe (Huang et al., 2010; Yaksi and Wilson, 2010). We examined these possibilities.

The MB feedback pathway does not seem to be involved, given that synaptic output from these neurons is not required during odorant exposure. Thus, exposure of MB247>UAS-Shits ${ }^{t s l}$ flies to $\mathrm{EB}$ at $32^{\circ} \mathrm{C}$, which blocks neurotransmission from these neurons, had no significant effect on habituation compared with genetic or temperature controls (Fig. 5A).

To test the potential involvement of the $\mathrm{PN}-\mathrm{eLN}$-inhibitory LN (iLN) pathway in habituation, we examined odor- and PN-induced habituation in $\operatorname{sh} a k B^{2}$ mutants in which electrical synapses between eLNs and iLNs are disrupted (Fig. 5B,C) (Huang et al., 2010; Yaksi and Wilson, 2010). Our observation that odor-exposed $s h a k B^{2}$ or temperature-exposed $\operatorname{shakB}^{2} / Y$; GH146Gal4/+;UAS TRPA1/+ flies are defective for habituation shows a role for electrical synapses in olfactory habituation and is consistent with a model in which recurrent inhibition onto PNs that is required for habituation is mediated via the $\mathrm{PN}-\mathrm{eLN}-\mathrm{LN} 1$ pathway.

\section{Discussion}

We conclude that PN excitation alone can drive plasticity in the antennal lobe, independently of OSNs. We propose that this occurs through potentiation of recurrent inhibition. This recurrent inhibitory pathway may be polysynaptic: e.g., PN-eLN-LN1-PN. However, the requirement for cAMP, GABA, and glutamate signaling components in LN1 neurons indicates that these inhibitory neurons, which synapse directly onto PNs (Tanaka et al., 2009), undergo plasticity required for habituation. Although inhibitory LN1s connect to a large fraction of PNs, we propose that a subset of inhibitory synapses onto active PNs are selectively strengthened during prolonged PN activity. The resulting synapse-specific enhancement of recurrent inhibition underlies odorant-selective olfactory habituation (Stopfer and Laurent, 1999; Bazhenov et al., 2005; Das et al., 2011).

The observation that $\mathrm{PN}$ activity, rather than OSN activity, is the driver for olfactory habituation is consistent with observations in other biological systems, where others have argued that it is useful to modulate a system's input sensitivity based on feedback from its functional output, rather than on levels of input (Alon, 2006). In the case of the olfactory system, the dynamic pattern of the odorant-evoked $\mathrm{PN}$ ensemble activity may provide a less noisy and more highly specified odor representation than the corresponding pattern of OSN activity (Laurent, 2002; Wilson and Mainen, 2006; Su et al., 2009; Gupta and Stopfer, 2011). Therefore, the distinctive pattern of feedback excitation from PNs onto LNs would more precisely specify a given odorant than the pattern of feedforward OSN excitation. In addition, a PN-LN pathway that allows OSNs to remain on-line after habituation makes it easier for attentional or emotional inputs to signal dishabituation. A PN-LN pathway for habituation is consistent with previous electrophysiological and/or calcium-imaging studies, which report plasticity of odor-evoked PN responses without corresponding changes in OSNs (Stopfer and Laurent, 1999; Devaud et al., 2001; Sachse et al., 2007). Not only does feedback from PNs initiate habituation (Fig. 1), but its potentiation at the step of LN-PN transmission underlies the reduced response of the olfactory system to the familiar odorant (Das et al., 2011).

Indeed, plasticity of inhibitory feedback, which allows the sensitivity levels of a system to be tuned in response to its recent history, appears as a frequent regulatory motif in the design of adaptive systems. Similar plasticity of feedback inhibition is used in many engineering and biological systems: for example, thermostats of room heaters, methylation in bacterial chemotaxis, and phophatase control of the MAPK kinase pathway (Bhalla et al., 2002; Alon, 2006).

A significant feature of our proposed mechanism for olfactory habituation is that it is theoretically transferrable to other systems. Unlike sensory neurons, which are unique in that they do 
not receive synaptic inputs, $\mathrm{PNs}$ are conceptually similar to principal neurons in other parts of the brain in terms of their role in information coding as well as their regulation by feedforward and recurrent inhibition. In much the same manner that an ensemble of PN activity is postulated to represent an odorant (Laurent, 2002; Wilson and Mainen, 2006; Su et al., 2009), ensembles of principal neurons activity are thought to represent more complex cognitive percepts (Buzsáki, 2010). Importantly, in both cases, the ensemble of active principal neurons turns on a corresponding pattern of inhibitory feedback mediated by GABAergic neurons (Isaacson and Scanziani, 2011).

We propose a synaptic learning rule to account for olfactory habituation in the antennal lobe: namely, that the LN1 presynapse is facilitated when its postsynapse is strongly depolarized at the same time the presynapse is active. There is evidence for such Hebbian potentiation of inhibitory synapses in other systems (Fischer and Carew, 1993; Bazhenov et al., 2005; Nugent et al., 2007; Fernandez et al., 2009; Rath et al., 2011). If this rule, which we propose for GABAergic synapses in the fly antennal lobe, were to be applied in other brain regions, then one would predict that persistent activity of a given ensemble of principal cells would result in synapse-specific strengthening of inhibitory inputs made onto these principal cells (Bazhenov et al., 2005; Das et al., 2011; Glanzman, 2011).

Habituation to continuous, unreinforced exposure occurs to either simple or complex percepts (Thompson and Spencer, 1966; Glanzman, 2009). We suggest that the selective potentiation of feedback inhibition onto persistently active principal neurons may be an elegant, frequently relied on solution for the ubiquitous but poorly understood phenomenon of habituation.

\section{References}

Alon U (2006) An introduction to systems biology: design principles of biological circuits. Boca Raton, FL: Chapman and Hall/CRC.

Bazhenov M, Stopfer M (2010) Forward and back: motifs of inhibition in olfactory processing. Neuron 67:357-358.

Bazhenov M, Stopfer M, Sejnowski TJ, Laurent G (2005) Fast odor learning improves reliability of odor responses in the locust antennal lobe. Neuron 46:483-492.

Bhalla US, Ram PT, Iyengar R (2002) MAP kinase phosphatase as a locus of flexibility in a mitogen-activated protein kinase signaling network. Science 297:1018-1023.

Buzsáki G (2010) Neural syntax: cell assemblies, synapsembles, and readers. Neuron 68:362-385.

Corfas G, Dudai Y (1989) Habituation and dishabituation of a cleaning reflex in normal and mutant Drosophila. J Neurosci 9:56-62.

Das S, Sadanandappa MK, Dervan A, Larkin A, Lee JA, Sudhakaran IP, Priya R, Heidari R, Holohan EE, Pimentel A, Gandhi A, Ito K, Sanyal S, Wang JW, Rodrigues V, Ramaswami M (2011) Plasticity of local GABAergic interneurons drives olfactory habituation. Proc Natl Acad Sci U S A 108:E646-E654.

Devaud JM, Acebes A, Ferrús A (2001) Odor exposure causes central adaptation and morphological changes in selected olfactory glomeruli in Drosophila. J Neurosci 21:6274-6282.

Engel JE, Wu CF (1996) Altered habituation of an identified escape circuit in Drosophila memory mutants. J Neurosci 16:3486-3499.

Fernandez PC, Locatelli FF, Person-Rennell N, Deleo G, Smith BH (2009) Associative conditioning tunes transient dynamics of early olfactory processing. J Neurosci 29:10191-10202.

Fino E, Yuste R (2011) Dense inhibitory connectivity in neocortex. Neuron 69:1188-1203.

Fischer TM, Carew TJ (1993) Activity-dependent potentiation of recurrent inhibition: a mechanism for dynamic gain control in the siphon withdrawal reflex of Aplysia. J Neurosci 13:1302-1314.

Glanzman DL (2009) Habituation in Aplysia: the Cheshire cat of neurobiology. Neurobiol Learn Mem 92:147-154.

Glanzman DL (2011) Olfactory habituation: fresh insights from flies. Proc Natl Acad Sci U S A 108:14711-14712.

Gupta N, Stopfer M (2011) Insect olfactory coding and memory at multiple timescales. Curr Opin Neurobiol 21:768-773.
Hu A, Zhang W, Wang Z (2010) Functional feedback from mushroom bodies to antennal lobes in the Drosophila olfactory pathway. Proc Natl Acad Sci U S A 107:10262-10267.

Huang J, Zhang W, Qiao W, Hu A, Wang Z (2010) Functional connectivity and selective odor responses of excitatory local interneurons in Drosophila antennal lobe. Neuron 67:1021-1033.

Isaacson JS, Scanziani M (2011) How inhibition shapes cortical activity. Neuron 72:231-243.

Kitamoto T (2001) Conditional modification of behavior in Drosophila by targeted expression of a temperature-sensitive shibire allele in defined neurons. J Neurobiol 47:81-92.

Kullmann DM, Lamsa KP (2011) LTP and LTD in cortical GABAergic interneurons: emerging rules and roles. Neuropharmacology 60:712-719.

Lai SL, Lee T (2006) Genetic mosaic with dual binary transcriptional systems in Drosophila. Nat Neurosci 9:703-709.

Lamsa KP, Heeroma JH, Somogyi P, Rusakov DA, Kullmann DM (2007) Anti-Hebbian long-term potentiation in the hippocampal feedback inhibitory circuit. Science 315:1262-1266.

Larkin A, Karak S, Priya R, Das A, Ayyub C, Ito K, Rodrigues V, Ramaswami M (2010) Central synaptic mechanisms underlie short-term olfactory habituation in Drosophila larvae. Learn Mem 17:645-653.

Laurent G (2002) Olfactory network dynamics and the coding of multidimensional signals. Nat Rev Neurosci 3:884-895.

Liu X, Krause WC, Davis RL (2007) GABAA receptor RDL inhibits Drosophila olfactory associative learning. Neuron 56:1090-1102.

Liu X, Buchanan ME, Han KA, Davis RL (2009) The GABAA receptor RDL suppresses the conditioned stimulus pathway for olfactory learning. J Neurosci 29:1573-1579.

Masse NY, Turner GC, Jefferis GS (2009) Olfactory information processing in Drosophila. Curr Biol 19:R700-R713.

Nugent FS, Penick EC, Kauer JA (2007) Opioids block long-term potentiation of inhibitory synapses. Nature 446:1086-1090.

Pulver SR, Pashkovski SL, Hornstein NJ, Garrity PA, Griffith LC (2009) Temporal dynamics of neuronal activation by Channelrhodopsin-2 and TRPA1 determine behavioral output in Drosophila larvae. J Neurophysiol 101:3075-3088.

Rankin CH, Abrams T, Barry RJ, Bhatnagar S, Clayton DF, Colombo J, Coppola G, Geyer MA, Glanzman DL, Marsland S, McSweeney FK, Wilson DA, Wu CF, Thompson RF (2009) Habituation revisited: an updated and revised description of the behavioral characteristics of habituation. Neurobiol Learn Mem 92:135-138.

Rath LC, Galizia G, Szyszka P (2011) Multiple memory traces after associative learning in the honey bee antennal lobe. Eur J Neurosci 34:352-360.

Rosenzweig M, Brennan KM, Tayler TD, Phelps PO, Patapoutian A, Garrity PA (2005) The Drosophila ortholog of vertebrate TRPA1 regulates thermotaxis. Genes Dev 19:419-424.

Sachse S, Rueckert E, Keller A, Okada R, Tanaka NK, Ito K, Vosshall LB (2007) Activity-dependent plasticity in an olfactory circuit. Neuron $56: 838-850$.

Stopfer M, Laurent G (1999) Short-term memory in olfactory network dynamics. Nature 402:664-668.

Su CY, Menuz K, Carlson JR (2009) Olfactory perception: receptors, cells, and circuits. Cell 139:45-59.

Tanaka NK, Ito K, Stopfer M (2009) Odor-evoked neural oscillations in Drosophila are mediated by widely branching interneurons. J Neurosci 29:8595-8603.

Thompson RF, Spencer WA (1966) Habituation: a model phenomenon for the study of neuronal substrates of behavior. Psychol Rev 73:16-43.

Wilson RI (2011) Understanding the functional consequences of synaptic specialization: insight from the Drosophila antennal lobe. Curr Opin Neurobiol 21:254-260.

Wilson RI, Mainen ZF (2006) Early events in olfactory processing. Annu Rev Neurosci 29:163-201.

Wu CL, Xia S, Fu TF, Wang H, Chen YH, Leong D, Chiang AS, Tully T (2007) Specific requirement of NMDA receptors for long-term memory consolidation in Drosophila ellipsoid body. Nat Neurosci 10:1578-1586.

Xia S, Miyashita T, Fu TF, Lin WY, Wu CL, Pyzocha L, Lin IR, Saitoe M, Tully $\mathrm{T}$, Chiang AS (2005) NMDA receptors mediate olfactory learning and memory in Drosophila. Curr Biol 15:603-615.

Yaksi E, Wilson RI (2010) Electrical coupling between olfactory glomeruli. Neuron 67:1034-1047. 